\title{
II.-On the Arseniates of Baryta, Lime, and Magnesia, and the Separation of Arsenic from other Elements.
}

\author{
By Frederick Field, M.R.I.A., F.C.S.
}

AlL chemists must have experienced the difficulty of separating
arsenic from other elements with which - it is associated. It is
scarcely necessary to say that the method usually adopted, is to
precipitate the arsenic as sulphide with the other metals which are
thrown down by sulphide of hydrogen from their acid solutions,
and subsequently digest the precipitate with the sulphides of
ammonium or potassium. Many other bodies, however, are re-
dissolved as well as the sulphide of arsenic, the separation of
which in this stage of the process becomes exceedingly perplexing
and difficult. Even in the earlier operations of the analysis, the
arsenic has to be entirely converted from arsenic acid into
arsenious acid, in order to insure its entire precipitation, and this
is usually effeted by is usually effected by sulphurous acid or a sulphite. When the
arsenic exists in a very large proportion, repeated deoxidations and precipitations are necessary, which not only involve the expenditure of much time and labour, but are attended with loss of the substance under analysis. Much of the arsenic, it is true, can be eliminated by calcination, with occasional additions of carbon; and probably in the establishments where nickel and cobalt are obtained on a large scale, eight or nine-tenths of the arsenic are got rid of by this method. Even then the manipulator finds it necessary to pass a powerful and long-continued stream of sulphuretted hydrogen through the solution of the oxides, and to expel the excess by ebullition, previous to the separation of iron, nickel, and cobalt. Calcination, however, is inadmissible in chemical analysis, for-independently of the mechanical loss which is likely to occur--some metals, especially silver, are volatile in arsenical fumes. Moreover, presuming that all the operations mentioned above, have been carried out successfully, the arsenic cannot be separated entirely by the alkaline sulphides. $\mathrm{Mr}$. Bloxam has shown* that when the proportion of copper is large,

\footnotetext{
* Quarterly Journal of the Chemical Society, vol. v, p. 104.
} 
much arsenic is left with its sulphide, even after long digestion with sulphide of ammonium, and indeed that 1 per cent. of arsenic becomes insoluble in the alkaline sulphide, when the remainder consists of sulphide of copper. In the analysis of the ores of nickel and cobalt, I have found the formation of arseniate of potash highly useful, as a means of separating arsenic from these metals. When the arseniates of nickel and cobalt, in solution of hydrochloric acid, are boiled with excess of potash, they are entirely decomposed, the whole of the arsenic acid uniting with the alkali. Ammonia cannot be employed, as the arseniate of nickel is very slowly decomposed by this reagent. When native arseniate of nickel is dissolved in hydrochloric acid, and ammonia added, a gelatinous white precipitate is produced, and the supernatant liquid is colourless; and in order to obtain the blue ammoniacal solution, it is first necessary to free the nickel from arsenic. Iron is also present in this class of minerals, and sesqui-arseniate of iron is highly soluble in ammonia, so that if the substance contain arsenic, it is impossible to separate nickel from iron by that alkali. When an arsenical ore of cobalt (containing iron) is digested in nitro-hydrochloric acid, and ammonia added in excess, no precipitate is formed, provided there be sufficient arsenic to convert the whole of the iron into arseniate, and chloride of ammonium to form a soluble compound with cobalt. The solution has a fine brown colour, and is decomposed by sulphate of magnesia, sesquioxide of iron and ammonio-arseniate of magnesia being formed. When only iron and arsenic are present, both are precipitated in this manner, and the supernatant liquid contains merely ammoniacal salts.

The employment of sulphate of magnesia in conjunction with ammonia, for the determination of arsenic acid, has been proposed by Levol;* and Rose, in his Handbook of quantitative analysis, strongly recommends this process as very accurate, provided certain precautions are adopted in preparing the arseniate of magnesia and ammonia for the determination of its weight. Rose also applies this method to the separation of arsenic from antimony, and states that it is the best process known, furnishing very accurate results, if conducted with care.

I was led in the first instance, without previous acquaintance with the results of Levol and Rose, to institute experiments with a view to the application of magnesia to the determination

*Ann. Ch. Phys, [3] xrii, 501. 
of arsenic in the above manner, and to submit to a comparative examination the precipitates produced by baryta, lime, and magnesia in an ammoniacal solution of arsenic acid, for the purpose of ascertaining the relative values of the alkaline earths in this branch of analysis. The present communication contains a summary of these experiments.

Arseniate of Baryta.-When chloride of barium is added to a solution of arsenic acid in ammonia, the latter being in excess, a copious precipitate of trisarseniate of baryta is formed, consisting of $3 \mathrm{BaO}, \mathrm{AsO}_{5}$. According to Graham, this substance attracts a small quantity of carbonic acid from the air. Berzelius says it is very slightly soluble in water, somewhat more soluble in aqueous ammonia; and Laugier informs us that the solubility does not seem to be increased by the presence of ammonia, potash or soda salts.* My own experiments differ considerably from the above.

The arseniate of baryta was found to consist, after very careful analysis, of-

or, $3 \mathrm{BaO}, \mathrm{AsO}_{5}$

$$
\begin{aligned}
& \text { Baryta . . 66.39 } \\
& \text { Arsenic Acid } 33.32 \\
& 99 \cdot 71
\end{aligned}
$$

Calculated.

$3 \mathrm{BaO}$. $66 \cdot 65$

Arsenic Acid 33.35

$100 \cdot 00$

The baryta was precipitated from the solution of the arseniate in hydrochloric acid, by sulphate of soda. After deoxidation by sulphurous acid, the arsenic was estimated as tersulphide-

$$
\text { 10.00 grs. } 3 \mathrm{BaO}, \mathrm{AsO}_{5}=6.639 \mathrm{BaO} \text { and } 3.55 \mathrm{AsS}_{3}=3.33 \mathrm{AsO}_{5} \text {. }
$$

$10.00 \mathrm{grs}$. arsenious acid were converted into arsenic acid, and ammonia added in excess. The addition of chloride of barium caused a precipitate, which, after washing with weak ammonia, until no chlorine could be detected in the filtrate, gave on desiccation at $300^{\circ}$ Fahr. -

$$
34.52 \text { grs. } 3 \mathrm{BaO}, \mathrm{AsO}_{5} \text {. } \quad 34.57 \text { grs. } 3 \mathrm{BaO}, \mathrm{AsO}_{5} \text {. Calculated. }
$$

Arseniate of baryta loses all its water at a temperature a little above $212^{\circ}$. After diying in a water-bath for several hours, 18.90 grs. only lost 0.05 on ignition. After drying upon a sand-

* Gmelin's Handbook, vol. iv, 304. 
bath, at a temperature which never exceeded $300^{\circ}$, in a platinum crucible, no loss was experienced on heating to redness.

With regard to the solubility of this salt, the following experiments were performed :

$10 \cdot 00$ grs. $3 \mathrm{BaO}, \mathrm{AsO}_{5}$ digested with 2000 grs. cold distilled water for forty-eight hours, lost $1 \cdot 10 \mathrm{gr}$. The filtrate gave $0.9 \mathrm{gr}$. sulphate of baryta on addition of sulphate of baryta.

10.00 id.digested with $2000 \mathrm{grs}$. solution of chloride of ammonium (containing 100 grs. of the dry salt) lost 3.852. The filtrate gave $3 \cdot 4.1$ sulphate of baryta.

$10.00 \mathrm{id}$. digested with solution of ammonia formed by adding 200 grs. ammonia, sp. gr. $\cdot 880$, to 1800 grs. water, lost $0 \cdot 06$.

Sulphate of soda hardly produced any precipitate of sulphate of baryta in the filtrate.

From this it appears, that, although very soluble in chloride of ammonium, and moderately so in water, arseniate of baryta is very insoluble in aqueous ammonia. And even (as in most cases in analysis) when chloride of ammonium is present, the addition of excess of ammonia prevents in a great measure the solubility of the baryta salt. And it may be mentioned here, that ammonia determines the insolubility both of the magnesia and lime arseniates in the same manner, precipitating the ammonioarseniates from their solution in chloride of ammonium. Chloride of barium can be advantageously employed for the detection of small quantities of arsenic acid, when in combination with copper and other metals.

100.00 grs. of copper free from sulphur were placed in a flask, together with 0.20 grs. arsenious acid. After solution in nitric acid, and addition of excess of ammonia, sulphate of magnesia caused a precipitate, which settled at the bottom of the vessel after 12 hours. On filtering the small precipitate, arsenic was readily detected by the usual test.

Minerals containing sulphur arsenic and nickel, can be very neatly and correctly analysed by the employment of a baryta salt. By adding chloride of barium in excess to the acid solution, sulphate of baryta is precipitated, and after filtration and addition of a large excess of ammonia, all the arsenic is precipitated, provided sufficient chloride of barium has been employed. It is necessary to add this reagent to the acid solution, and therefore to decompose the arseniate of nickel in hydrochloric acid. There is no fear of any nickel remaining with the 
arseniate of baryta, which is a dense white powder, and can be thoroughly and expeditiously washed. For this purpose, of course a weak solution of ammonia is employed, instead of pure water.

Arseniate of Lime and Ammonia.-When diarseniate of ammonia, potash, or soda is added to a solution of chloride of calcium, trisarseniate of lime is formed, and the supernatant liquid becomes acid.

When chloride of ammonium, trisarseniate of ammonia, and lime-water are mixed together, crystals of arseniate of ammonia and lime $2 \mathrm{CaO}, \mathrm{NH}_{4} \mathrm{O}, \mathrm{AsO}_{5}$ are found.* $\mathrm{I}$ have met with two distinct precipitates during the course of my experiments, which possessed the following characteristics :

10.00 grs. arsenious acid were converted into arsenic acid, ammonia added in very slight excess, and subsequently solution of chloride of calcium. The precipitate, after prolonged washing with water and dessication at $300^{\circ}$, weighed 15.50 grs., and on ignition lost only $0.80 \mathrm{gr}$, yielding 14.70 of a compound of arsenic acid and lime. A large quantity of arsenic was detected in the filtrate.

10.00 grs. of arsenious acid were similarly treated, and the preci. pitate washed with weak ammonia; after drying at $300^{\circ}$, it was found to weigh $18 \cdot 24$ grains, and $17 \cdot 02$ on ignition. The precipitates in both instances seemed to consist of different substances, one of a very slightly crystalline powder, the other of tolerably large needles, united together in stellated masses. Although in the latter experiment the $10 \mathrm{grs}$. of arsenious acid yielded 17.02 of lime salt, and by calculation it should yield $17 \cdot 10$ diarseniate of lime $\left(2 \mathrm{CaO}, \mathrm{AsO}_{5}\right)$ the loss by ignition was so small, and so much less than it would have been, had an atom of ammonia been expelled, as to lead me to doubt very much if the precipitate were the arseniate of lime and ammonia, notwithstanding that it evolved much ammonia on ebullition with potash.

In order to analyze the residue left upon ignition, advantage was taken of the fact, that arseniate of lime is perfectly decomposed by a boiling solution of oxalate of ammonia.

It is better, however, to dissolve the arseniate in hydrochloric acid, add the oxalate, and finally excess of ammonia, and boil for some time. The whole of the arseniate is found in the filtrate, and the lime in the precipitate as oxalate of lime.

The 14.70 grains boiled in this manner gave, on ignition, 8.00 carbonate of lime $=5.48$ lime, and the $17.02 \mathrm{grs}$. gave

* Gmelin's Handbook, vol. iv, p. 304. 
ARSENIATES OF LIME, BARYTA, AND MAGNESIA.

$11.56 \mathrm{CaO}, \mathrm{CO}_{2}=6.47 \mathrm{CaO}$. Sulphate of magnesia was added to the filtrate, and after standing some time, the ammonio-magnesian arseniate collected, and the arsenic estimated.

Composition of Diarseniate of Lime, $2 \mathrm{CaO}, \mathrm{AsO}_{5}$

Calculated.

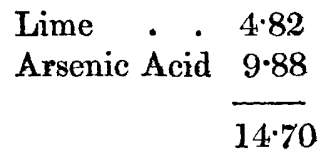

Lime . $5 \cdot 48$

Arsenic Acid $9 \cdot 27$

Lime . . $5 \cdot 57$

Arsenic Acid 11.45

Lime . . 6.47

Arsenic Acid 10.44

$17 \cdot 02$

$16 \cdot 91$

It is evident from the above analyses, that the salt under consideration is not diarseniate of lime, but probably a mixture of diarseniate and triarseniate, the former produced by the decomposition of the ammonia compound, thrown down from the original solution in company with the triarseniate. The loss by ignition, as well as the quantities found of arsanic acid and lime leave no doubt that this is the case.

$3 \mathrm{CaO}, \mathrm{AsO}_{5}+2 \mathrm{CaO}, \mathrm{NH}_{4} \mathrm{O}, \mathrm{AsO}_{5}$, upon ignition $=3 \mathrm{CaO}, \mathrm{AsO}_{5}+$ $2 \mathrm{CaO}, \mathrm{AsO}_{5}$.

And the relative numbers are as follows:

$$
\begin{array}{rllll} 
& & & \text { Found. } \\
\text { As } 396: 370:: 18 \cdot 24: 17 \cdot 04 & \ldots & 17 \cdot 02 \\
\text { „ } 396: 370:: 15 \cdot 50: 14 \cdot 47 & \ldots & 14 \cdot 70
\end{array}
$$

And with regard to the quantities of lime and arsenic acid: $3 \mathrm{CaO}, \mathrm{AsO}_{5}+2 \mathrm{CaO}, \mathrm{AsO}_{5}$,

Calculated.

Lime . . 5.56

Arsenic Acid 9·14

$$
\overline{14 \cdot 70}
$$

Lime . . 6.44

Arsenic Acid 10.58
Found.

Lime . 5.48

Arsenic Acid 9.27

14.75

Lime . 6.47

Arsenic Acid 10.44 
It is not at all surprising that when ammonia is not in great excess, that trisarseniate of lime should be formed, as even diarseniate of ammonia produces this compound. It is, however, rather singular, that, when in combination with the diarseniate of lime, the trisarseniate does not lose arsenic acid upon ignition, which it does when heated per se.*

The arseniate of lime and ammonia can be readily formed by adding ammonia and trisarseniate of ammonia to a solution of chloride of calcium. The precipitants must be in considerable excess. This salt crystallises from weak solutions in large needleshaped crystals, and from more concentrated solutions, it separates as a white crystalline mass. Like the baryta compound, it is more soluble in water than in aqueous ammonia, and soluble to a great extent in chloride of ammonium. It retains an atom of water at $212^{\circ}$, and consists at that temperature of

$$
2 \mathrm{CaO}, \mathrm{NH}_{4} \mathrm{O}, \mathrm{AsO}_{5}+\mathrm{HO} \text {. }
$$

10.00 grs. in 2000.00 grs. water, lost 0.40 grs.

10.00 grs. " $"$ grs. weak ammonia, lost 0.02 grs.

10.00 grs. ", ", grs. chloride of ammonium, containing 100 grs. dry salt, lost $8 \cdot 300 . \dagger$

This salt loses its atom of water at a temperature a little beyond $212^{\circ}$.

Dried at $280^{\circ}$.

$18 \cdot 25$

$11 \cdot 90$

$13 \cdot 20$

on ignition gave

Found. Calculated.

$\begin{array}{ll}15 \cdot 80 & 15 \cdot 83 \\ 10 \cdot 25 & 10 \cdot 32 \\ 11 \cdot 48 & 11 \cdot 45\end{array}$

The residues were analysed separately, and found to consist of $2 \mathrm{CaO}, \mathrm{AsO}_{5}$, as the following numbers will show :--

\begin{tabular}{lrr} 
Calculated. & Found. \\
Lime & $5 \cdot 18$ & $5 \cdot 01$ \\
Arsenic Acid & $10 \cdot 62$ & $10 \cdot 49$ \\
\hline $15 \cdot 80$ & & $15 \cdot 50$
\end{tabular}

* The cold solution of this salt in chloride of ammonium, evolves ammonia copiously on ebullition, with formation of diarseniate of lime, which still remains dissolved in the liquid. According to Wach, diarseniate of lime when in solution of ammoniacal salts, becomes converted into arseniate of lime and ammonia, but this is decomposed on boiling with a re-formation of diarseniate of lime.

tThe trisarseniate of lime is decomposed when ignited alone (Simon), Gmelin's Handbook, vol. iv, p. 304. 
ARSENIATES OF LIME, BARYTA, AND MAGNESIA.

\begin{tabular}{lcc}
\multicolumn{2}{c}{ Calculated. } & Found. \\
Lime . . & $3 \cdot 41$ & $3 \cdot 38$ \\
Arsenic Acid & $6 \cdot 84$ & $6 \cdot 75$ \\
\cline { 2 - 2 } & $10 \cdot 25$ & $10 \cdot 13$ \\
Lime . . & $3 \cdot 76$ & $3 \cdot 64$ \\
Arsenic Acid & $7 \cdot 72$ & $7 \cdot 69$ \\
\cline { 2 - 3 } & $\frac{11 \cdot 48}{11 \cdot 33}$
\end{tabular}

Arseniate of Magnesia and Ammonia.-This salt is thrown down in great purity, when arseniate of ammonia and excess of ammonia are added to a soluble salt of magnesia.

In applying this salt to the estimation of arsenic, Levol recommends its ignition, and the determination of the arsenic as pyroarseniate of magnesia $\left(2 \mathrm{MgO}, \mathrm{AsO}_{5}\right)$. But Rose has shown that a portion of arsenic acid is reduced, at a high temperature by ammonia, and volatilised, so that a loss in weight due to that reaction, is sustained in proportion to the necessary duration of the ignition. It is stated by the latter chemist that the weight of the double salt may be determined either by its desiccation in vacuo, in which case its composition is represented by the formula $2 \mathrm{MgO}, \mathrm{NH}_{4} \mathrm{O}, \mathrm{AsO}_{5}+12 \mathrm{HO}$, or at $212^{\circ} \mathrm{F}$., when it consists of-

$$
2 \mathrm{MgO}, \mathrm{NH}_{4} \mathrm{O}, \mathrm{AsO}_{5}+\mathrm{HO} \text {. }
$$

Like the corresponding lime salt, it loses its water at a slight increase of temperature; at $180^{\circ} \mathrm{F}$. it retains three atoms of water, and it looses its ammonia between $500^{\circ}$ and $600^{\circ}$.

Its formula may be expressed at various temperatures, as follows :-

\begin{tabular}{|c|c|c|c|}
\hline Wher & dried & in vacuo, & $2 \mathrm{MgO}, \mathrm{NH}_{4} \mathrm{O}, \mathrm{AsO}_{5}+12 \mathrm{HO}$ \\
\hline From & $180^{\circ}$ & $200^{\circ}$ & $\mathrm{H}_{4} \mathrm{O}, \mathrm{AsO}_{5}+3 \mathrm{HO}$ \\
\hline At & 212 & $\ldots$ & $2 \mathrm{MgO}, \mathrm{NH}_{4} \mathrm{O}, \mathrm{AsO}_{5}+\mathrm{HO}$ \\
\hline At & 300 & & $2 \mathrm{MgO}, \mathrm{NH}_{4} \mathrm{O}, \mathrm{AsO}_{5}$ \\
\hline At & 600 & . & $2 \mathrm{MgO}, \mathrm{AsO}_{5}$. \\
\hline
\end{tabular}

In the estimation of arsenic from this compound, it is therefore very necessary to be extremely cautious in its desiccation. Specimens dried upon a filter placed above a sand-bath, with a thermometer suspended at the same distance from the heated surface (the mercury never rising above $300^{\circ}$ ), were found to have lost the whole of their water in from four to five hours. 
The following experiments were tried regarding the solubility of this double salt :-

10.00 grs. digested with 2000 grs. water, lost 0.28 grs. $10 \cdot 00$ $10 \cdot 00$

",

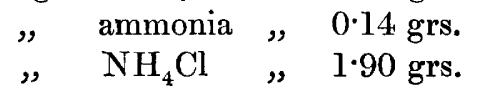

Ammonia precipitates the salt from its solution in chloride of ammonium.

The following diagram will give, in a tabular form, the solubility of the arseniates of lime and ammonia, magnesia and ammonia, and arseniate of baryta, in water, ammonia, and chloride of ammonium.

\begin{tabular}{|c|c|c|c|c|c|c|c|}
\hline \multirow[b]{2}{*}{ Water } & \multicolumn{3}{|c|}{$\begin{array}{c}10 \text { grs. } \\
2 \mathrm{MgO}, \mathrm{NH}_{4} \mathrm{O}, \mathrm{AsO}_{5}, \mathrm{HO} .\end{array}$} & \multicolumn{3}{|c|}{$\begin{array}{c}10 \text { grs. } \\
2 \mathrm{CaO}, \mathrm{NH}_{4} \mathrm{O}, \mathrm{AsO}_{5}, \mathrm{HO} .\end{array}$} & \multirow{2}{*}{$\begin{array}{c}10 \text { grs. } \\
3 \mathrm{BaO}, \mathrm{AsO}_{5} . \\
2000 \ldots \\
1 \cdot 10\end{array}$} \\
\hline & 2000 & .. & $0 \cdot 28$ & 2000 & .. & 0.40 & \\
\hline Ammonia... & 2000 & .. & $0 \cdot 14$ & 2000 & .. & 0.02 & $2000 \ldots 0.06$ \\
\hline $\begin{array}{c}\text { Chloride of } \\
\text { Ammonium }\end{array}$ & 2000 & .. & $1 \cdot 90$ & 2000 & .. & 830 & $2000 \ldots 3 \cdot 85$ \\
\hline
\end{tabular}

From the foregoing experiments, the relative advantages and disadvantages in the employment of the salts of the alkaline earths may be estimated, and-

1. The precipitation of arsenic acid by a soluble salt of lime.

This is disadvantageous when sulphuric acid is present in the liquid. Sulphate of lime would be precipitated, and require long and protracted washing for its entire separation. Neither could the arseniates be freed from the sulphate by dilute hydrochloric acid, as the latter is especially soluble in that menstruum. Another objection is, the uncertainty of the compound precipitated, which would render its analysis indispensable when the quantity of arsenic, as well as its abstraction from other bodies, is desired. On the other hand, its great insolubility in ammonia, renders the employment of a lime-salt, in certain circumstances, very advantageous.

2. The employment of a soluble baryta salt. The objection regarding the sulphuric acid, cannot be urged, as in the case of the lime-salt. Sulphate of baryta is easily separated from its arseniate, and indeed the whole of the sulphuric acid could be 
removed by chloride of barium, previously to the introduction of the ammonia. When chloride of ammonium does not exist in any very great quantity in the solution, the employment of baryta may be resorted to advantageously.

3. By a magnesia salt. Magnesia possesses many advantages over lime and baryta, which can be easily appreciated in practice. The ammonia-magnesian arseniate, after standing some hours, settles down in a heavy crystalline mass, so hard and dense, that the supernatant liquid may be frequently decanted off, and the crystals drained, thus rendering the subsequent washing very expeditious and easy. There is no fear of sulphuric acid being in the precipitate, which possesses the advantage of being very definite in composition, and insoluble in weak ammonia. From my own experience I should recommend magnesia above baryta and lime, if it were only for the greater facility of its management in chemical analysis.

The following estimations were invariably made with a magnesian salt :-

10.00 gr. arsenious acid converted into arsenic acid, and precipitated with a magnesian salt, gave $18.872 \mathrm{MgO}, \mathrm{NH}_{4} \mathrm{O}$, $\mathrm{AsO}_{5}+\mathrm{HO}$. Calculation $19 \cdot 19$.

10.00 gr. gave 18.94 .

$10.00 \mathrm{gr} . \mathrm{AsO}_{3}$, and $1 \mathrm{gr}$. copper dissolved in nitric acid, gave $19 \cdot 142 \mathrm{MgO}, \mathrm{NH}_{4} \mathrm{O}, \mathrm{AsO}_{5}+\mathrm{HO}=9 \cdot 97 \mathrm{AsO}_{3}$.

1.00 gr. $\mathrm{AsO}_{3}+10.00 \mathrm{gr}$. Cu, gave $1.902 \mathrm{MgO}, \mathrm{NH}_{4} \mathrm{O}, \mathrm{AsO}_{5}+\mathrm{HO}$; and when $0.19 \mathrm{gr} . \mathrm{AsO}_{3}=0.076$ arsenic was boiled with $\mathrm{NO}_{5}$, and $100 \cdot 00$ of copper, a crystalline deposit of the ammonia-magnesian arseniate was observed on the sides of the flasks, after standing for 24 hours. On drawing off the copper and washing the crystals with weak ammonia, they were dissolved in $\mathrm{HCl}$, and a clear yellow pentasulphide of arsenic obtained, on addition of a few drops of sulphide of ammonium, and afterwards, excess of hydrochloric acid.

It has been before observed that nickel can be easily and perfectly separated from arsenic by the introduction of a magnesian salt. In the following experiments, with a given weight of ore (?), the arsenic was not estimated, as the ore contained iron which was precipitated with the ammonio-magnesian arseniates.

The filtrate was precipitated by sulphide of ammonium, the sulphide dissolved, and the nickel thrown down as oxide by potash 
$\begin{array}{ccccc}\text { The first experiment gave } & \ldots & 6 \cdot 127 & \text { oxide of nickel. } \\ \text { second ", } & " & 6 \cdot 00 & , \\ \text { third ", } & " & 5 \cdot 95\end{array}$

The Separation of Arsenic from Antimony by means of a soluble magnesian salt in the presence of ammonia, is very strongly recommended by Rose; and my own experiments fully confirm the statements of that chemist. The method described in detail in his Handbook succeeds perfectly; I may state, however, that as the tartaric acid, added to the solution of the oxidised metals for the purpose of retaining the antimony in solution, is sometimes liable to occasion the separation, with the arseniate, of a small quantity of tartrate of magnesia and ammonia, I have found it advisable to substitute sulphate of ammonia for the chloride of ammonium added previous to the ammonia. The double tartrate is very readily soluble, and the double arseniate particularly in. soluble in the sulphate of ammonia.

The following analyses will show the correctness of the method :-

Taken.

Arsenious Acid 5.00 Antimony . $5 \cdot 00$

Arsenious Acid $5 \cdot 00$ Antimony . $5 \cdot 00$

$$
\begin{gathered}
2 \mathrm{MgO}, \mathrm{NH}_{4} \mathrm{O}, \mathrm{AsO}_{5}+\mathrm{HO} \quad 9 \cdot 54=4.97 \mathrm{AsO}_{3} . \\
\text { " } \quad 9 \cdot 52=4.95 \mathrm{AsO}_{3} .
\end{gathered}
$$

The antimony was not determined in the above analyses; in those below, the arsenic was estimated as before, sulphide of ammonium added in excess to the filtrate, until the sulphide of antimony was completely re-dissolved. It was afterwards precipitated by hydrochloric acid. The precipitate was dried at $212^{\circ}$, weighed, and a portion examined quantitatively for sulphur, the weight of which was deducted, and the quantity calculated from the whole precipitate.

Taken.

Found.

$$
\begin{array}{rlrlr}
\text { Arsenious Acid . } 10.00 & =\text { Arsenic } 7 \cdot 57 & \ldots & 7 \cdot 39 \\
\text { Antimony . . } & =\frac{6 \cdot 80}{14 \cdot 37} & & \frac{7 \cdot 04}{14 \cdot 43} \\
\text { Arsenious Acid . } 12 \cdot 40 & =\text { Arsenic } 9.39 & \ldots & 9 \cdot 12 \\
\text { Antimony . . . } & =\frac{12 \cdot 40}{21 \cdot 79} & & \frac{12 \cdot 23}{21 \cdot 35}
\end{array}
$$


ARSENIATES OF LIME, BARYTA, AND MAGNESIA.

Taken.

Arsenious Acid $1.00,=$ Arsenic $0.75 \quad$ Found

Antimony $. \cdot .=\frac{10.00}{10 \cdot 75} \quad \cdots \quad \frac{9.89}{10.57}$

Arsenious Acid 10.00 . = Arsenic 7.57 $\quad . \quad 7.42$

Antimony . . . = $\quad 1.00 \quad \ldots \quad 0.98$

$\overline{8.57} \quad \ldots \quad \overline{8 \cdot 40}$

Arsenious Acid 0.10 gave a crystalline precipitate of Altimony $\quad 5 \cdot 00\} 2 \mathrm{MgO}, \mathrm{NH}_{4} \mathrm{O}, \mathrm{AsO}_{5}+\mathrm{HO}$ on the

Arsenious Acid 5.00 7 filtrate gave an orange-red preciAntimony . 0.10\} pitate of sulphide of antimony.

Arsenic cannot be separated from tin by means of magnesia. Ammonia produces no precipitate in the solution of the bichloride of tin when tartaric acid is present, but a precipitate is produced by the introduction of magnesian salts, which appears to contain both tin and magnesia, but which, so far as I know, has not been examined. Hopes may be entertained that antimony may be separated from tin by taking advantage of this fact.

The subject is now under consideration, and promises satisfactory results, though rather difficult and laborious. 